\title{
Avances en la regulación y control del estado térmico del horno alto ${ }^{(\bullet)}$
}

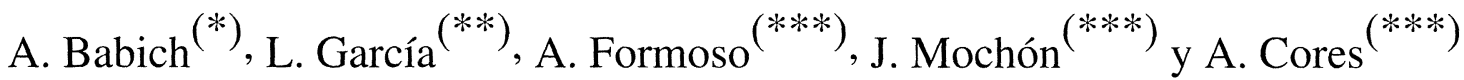

Resumen utiliza un sistema automatizado de dirección (SAD) para el control del estado térmico del crisol
del horno alto, teniendo en cuenta la relación que existe entre la temperatura del arrabio y su
contenido de silicio. Por la magnitud de la exergía térmica del proceso, que determina la capacidad
de trabajo del calor, se pueden valorar las variaciones de calentamiento del crisol y realizar las
acciones necesarias de control y regulación, como son las variaciones en la inyección de carbón
pulverizado y en la relación sinterizado/coque en el lecho de fusión del horno alto.

Palabras clave: Estado térmico del crisol. Inyección de carbón pulverizado. Relación sinterizado/coque. Control en línea. Automatización avanzada.

\section{Advances in the regulation and control in the thermal state of the blast furnace}

\begin{abstract}
An automated system of direction (SAD) for the control of the thermal state of the hearth of the blast furnace is used, taking into account the interrelation existing between the pig iron temperature and its silicon content. By the magnitude of the thermal exergy of the process, which determines the heat work capacity the variations in the heating of the hearth can be valued and carried out the necessary control and regulation actions, such as the variations in the pulverized coal injection and in the sinter/coke relation in the melting bed of the blast furnace.
\end{abstract}

Keywords: Thermic state in the hearth. Pulverized coal injection. Relation sinter/coke. Control on line. Advanced automation.

\section{INTRODUCCIÓN}

Han sido numerosos los intentos de crear un modelo universal único para el control de la marcha del horno alto; sin embargo, hasta el momento, esto no se ha conseguido por no disponer de un modelo matemático que se ajuste adecuadamente al proceso. Actualmente, entre las vías con futuro para automatizar la operación del horno alto, se encuentran la aplicación de sistemas de lógica difusa y de

$(\bullet)$ Trabajo recibido el día 6 de junio de 1996.

(*) Universidad Estatal Tecnológica de Donetsk. Arteoma, 58. 340066 Donetsk (Ucrania).

(**) Unión de Empresas de Recuperación de Materias Primas. $1^{a}$ Avda., 1604. 11300 Ciudad de La Habana (Cuba).

(***) Centro Nacional de Investigaciones Metalúrgicas (CSIC). Avda. de Gregorio del Amo, 8. 28040-Madrid (España). redes neuronales artificiales ( 1 y 2 ). Otra vía de interés es la utilización de sistemas automatizados de dirección (SAD) locales, útiles tanto para el control de los procesos locales de la fusión, como para ejecutar posteriormente interconexiones multifuncionales jerarquizadas con estructuras descentralizadas. Los sistemas creados tomando como base principios jerarquizados encuentran cada vez mayores aplicaciones, debido a la facilidad con que es posible implementar SAD basados en microcomputadores (3).

Para crear sistemas que permitan controlar el estado térmico del horno alto es necesario disponer de parámetros reguladores del proceso, datos operativos sobre el estado térmico del horno, algoritmos de control y técnicas de computación.

En este trabajo se investiga el control del estado térmico del horno alto mediante la implantación de un sistema SAD como modelo de control. 


\section{INVESTIGACIÓN Y SELECCIÓN DE PARÁMETROS REGULADORES DEL PROCESO}

La regulación del estado térmico del horno alto en las zonas superiores da lugar a notables retardos en el tiempo, debidos a la velocidad de descenso de los materiales de la carga, según la altura del horno, y a la propia inercia de los procesos térmicos. La regulación en las zonas superiores debe realizarse en combinación con las acciones en las zonas inferiores, ya que éstas no están sometidas a retardos y los efectos en su control son más inmediatos. Todas las acciones reguladoras en la parte inferior del horno son efectivas para controlar el calentamiento del crisol, salvo con aquellos combustibles auxiliares (gas natural y fuel oil) que se inyectan con el viento.

$\mathrm{Al}$ estudiar las características dinámicas del horno, en función de los combustibles inyectados, se ha determinado que la influencia de estos, al variar sus caudales de inyección, es compleja y su acción sobre el contenido de silicio del arrabio (Fig. 1a)) se manifiesta de dos maneras. Inicialmente, éste tiende a disminuir y después aumenta. Experimentalmente, se ha comprobado que el efecto completo de regulación se alcanza después de 12-18 h. El carácter complejo de los procesos transitorios al variar los caudales de inyección de gas natural y fuel oil está relacionado, en particular, con la disociación de grandes cantidades de hidrógeno.

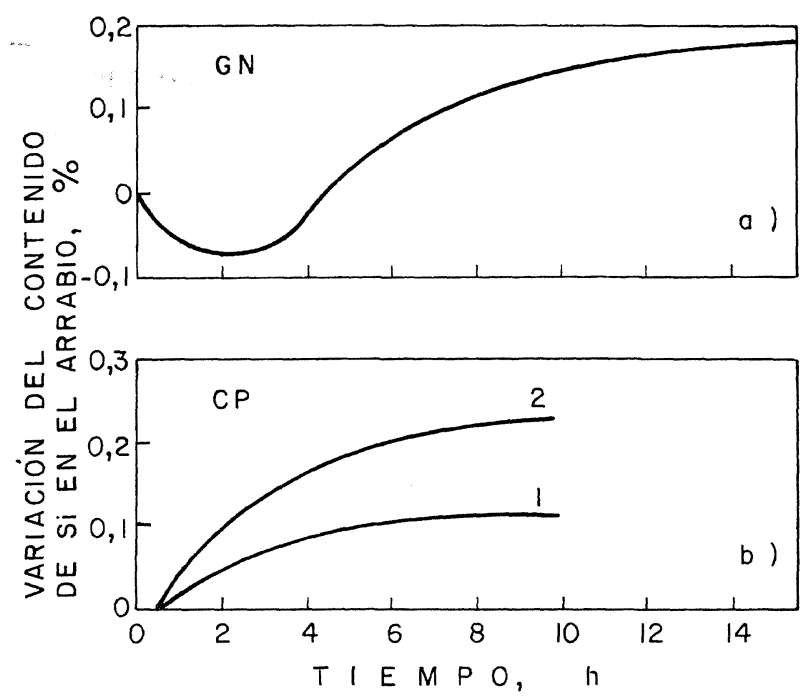

FIG. 1.- Dinámica de la variación del contenido de silicio en el arrabio mediante la regulación del estado térmico del crisol. a) Inyección de $1.000 \mathrm{~m}^{3} / \mathrm{h}$ de gas natural. b) Inyección de carbón pulverizado $(1: 1 \mathrm{t} / \mathrm{h} ; 2: 2 \mathrm{t} / \mathrm{h})$.

FIG. 1.- Variation dynamics in the pig iron silicon content by the thermal state regulation in the hearth. a) $1,000 \mathrm{~m}^{3} / \mathrm{h}$ natural gas injection. b) Pulverized coal $(1: 1 \mathrm{t} / \mathrm{h} ; 2: 2 \mathrm{t} / \mathrm{h})$ injection
La inyección de carbón pulverizado (ICP) por toberas, de composición química similar a la del coque, no provoca variaciones considerables ni en la composición ni en el volumen de gases en las toberas. Por tanto, la influencia de su tasa de inyección en el contenido de silicio en el arrabio (Fig. 1b)) tiene lugar por un principio más simple. La ecuación que expresa la variación del contenido de silicio en el arrabio, al variar la tasa de inyección de carbón pulverizado (ICP) tiene la forma (4):

$$
\Delta[\mathrm{Si}]=0,0085 \Delta \mathrm{S}_{2}\left(1-\mathrm{e}^{-(\tau-0,5) / 3,5)}\right.
$$

donde:

$\Delta[\mathrm{Si}]=$ Variación del contenido de silicio en el arrabio, \%.

$\Delta \mathrm{S}_{2}=$ Variación de la tasa ICP, $\mathrm{kg} / \mathrm{t}$ de arrabio.

$\tau=$ tiempo, $\mathrm{h}$.

El análisis de la fórmula [1] muestra que el efecto completo de la regulación para un tiempo constante $(\tau=3,5 \mathrm{~h})$ se alcanza prácticamente en unas 8-9 h. En este caso, al inyectar CP, el contenido de silicio en el arrabio varía en una media de $0,085 \%$ por cada $10 \mathrm{~kg} \mathrm{CP} / \mathrm{t}$ de arrabio.

Las investigaciones realizadas han permitido elaborar y utilizar en los hornos altos de la planta siderúrgica de Donetsk (Ucrania) un método de control térmico del crisol, mediante la inyección de CP. En la figura 2 se incluye un ejemplo de control del régimen térmico de fusión con utilización de CP.

En la figura 2a) se aprecia que cuando la relación aglomerado/coque $(\mathrm{A} / \mathrm{C})$ en la carga del horno se reduce de 3,08 a 3,02 t/t, mediante la conveniente disminución del consumo de aglomerado (principalmente sínter), la temperatura del arrabio es de $1.450{ }^{\circ} \mathrm{C}$, con un contenido de silicio del $0,45 \%$. Tanto durante el período analizado como durante los días anteriores no se varían otros parámetros del viento. Una hora después, se inicia la ICP en una cantidad de 2,25 t/h hasta alcanzar un caudal de 4 $\mathrm{t} / \mathrm{h}$. En estas condiciones, se mantiene la relación $\mathrm{A} / \mathrm{C}$ en 3,02 t/t. Como resultado de la ICP, la temperatura del arrabio se eleva en $40-50{ }^{\circ} \mathrm{C}$ y el contenido de silicio en el arrabio, en $0,5 \%$.

En la figura $2 b$ ) se observa que, en el momento de la colada, que termina $17 \mathrm{~h}$ después, la temperatura del arrabio alcanza los $1.530{ }^{\circ} \mathrm{C}$ con un contenido de silicio del $1,52 \%$. En la colada siguiente, la temperatura del arrabio se mantiene en $1.530{ }^{\circ} \mathrm{C}$ y el contenido de silicio alcanza el 1,64\%. Transcurridas $19 \mathrm{~h}$, la relación aglomerado/coque aumenta a 3,23 t/t, mediante el aumento de la correspondiente cantidad de aglomerado. Al mismo tiempo, se deja de inyectar CP, cuyo caudal era de $5 \mathrm{t} / \mathrm{h}$. A continuación, el horno opera durante $8 \mathrm{~h}$ sin ICP, y con una elevada relación A/C. Como 

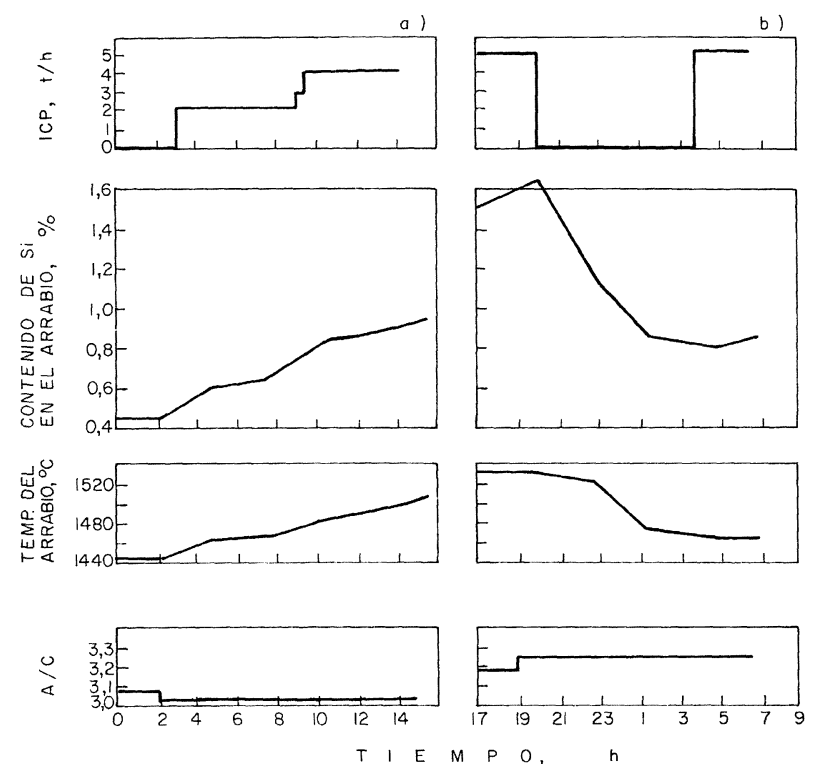

FIG. 2.- Control del régimen térmico del crisol con utilización de carbón pulverizado (CP) y la relación aglomerado/coque (A/C). a) Primer período de experimentación. b) Segundo período de experimentación.

FIG. 2.- Thermal rate control in the hearth by pulverized coal $(P C)$ utilization and the agglomerate/coke $(A / C)$ relation. a) First period of experimentation. b) Second period of experimentation.

resultado de no inyectar $\mathrm{CP}$ y de disminuir la cantidad de coque en la relación $\mathrm{A} / \mathrm{C}$, la temperatura del arrabio desciende a $1.460{ }^{\circ} \mathrm{C}$ y el contenido de silicio se reduce a $0,8 \%$. Posteriormente, se restablece la ICP.

Por tanto, la rápida respuesta de la ICP y el carácter simple del proceso transitorio ofrece ventajas en el caso de que sea necesario regular el estado térmico del crisol. La correspondencia en magnitud y tiempo entre las variaciones de la tasa de ICP y la relación de materiales en la carga garantiza el control óptimo del régimen térmico de fusión (5).

Entre otras acciones de control en la zona baja del horno, hay que tener en cuenta también el empleo de vapor de agua. La moderna tecnología de inyección de $\mathrm{CP}$, se debe acompañar con la inyección de vapor de agua en el viento para mantener el nivel requerido en la temperatura adiabática de llama.

\section{FUNDAMENTOS Y SELECCIÓN DE LOS CRITERIOS PARA DETERMINAR EL ESTADO TÉRMICO DEL HORNO ALTO}

El estado térmico del horno alto está interrelacionado con procesos de intercambio térmico y de masas, lo que dificulta la selección de parámetros que permitan su caracterización de forma satisfactoria.

En un horno alto en operación, el estado térmico del horno se determina por el contenido de silicio en el arrabio. Sin embargo, una vez concluida la colada, los resultados de los análisis químicos se demoran considerablemente, lo cual dificulta el control del estado térmico del horno. Además, el contenido de silicio en el arrabio no es un parámetro que determine unilateralmente el estado térmico, ya que su magnitud también depende de la basicidad de la escoria y de otra serie de factores.

En su conjunto, la composición química y la temperatura de los productos líquidos de la fusión caracterizan bastante bien el estado térmico del crisol del horno; sin embargo, hasta hace poco no existían medios adecuados para medir la temperatura del arrabio en continuo.

Últimamente, en la industria de equipos de control, se han desarrollado monitores fotopirométricos que, con una exactitud similar a la de los termopares, garantizan la medida de las temperaturas del arrabio y de la escoria durante la colada.

Estos equipos están construidos tomando como base modificaciones en la relación espectral e irradiación parcial de los fotodiodos, para que trabajen en un limitado rango espectral de longitud de onda. Dichos equipos son sistemas seguros y simples para el control automático de las temperaturas del arrabio y de la escoria a medida que sean colados del horno.

En este caso, para la medida automática en continuo de la temperatura del arrabio durante la colada, se ha utilizado un sensor fotopirométrico que controla la temperatura de la superficie de los cuerpos calientes según el calor que irradian. El margen de error en el registro de este equipo fue de 1,0-1,5\%.

Las informaciones sobre la temperatura y el contenido de silicio en el arrabio pueden utilizarse en el SAD cuando entre los calentamientos físico y químico del arrabio existe una relación fiable.

El cálculo termodinámico de la reducción del silicio en el horno alto, según la reacción en la que la sílice contenida en la escoria $\left(\mathrm{SiO}_{2}\right)$ se reduce a silicio que se incorpora al arrabio [Si], expresada como $\left(\mathrm{SiO}_{2}\right)+2 \mathrm{C}=[\mathrm{Si}]+2 \mathrm{CO}$, en presencia de hierro metálico, permite obtener la expresión siguiente para determinar la temperatura del arrabio:

$$
T_{\mathrm{a}}=\frac{571.222}{K-80,451 \lg \left(\frac{\mathrm{CaO}}{\mathrm{SiO}_{2}}\right)-19,155 \lg [\mathrm{Si}]}-273
$$

donde:

$T_{\mathrm{a}}=$ Temperatura del arrabio, ${ }^{\circ} \mathrm{C}$.

$K=$ Coeficiente, que depende de la presión parcial del $\mathrm{CO}$ en el crisol del horno (para $\left.P_{\mathrm{CO}}=98 \mathrm{kPa}, K=361,58\right)$.

$[\mathrm{Si}]=$ Contenido de silicio en el arrabio, $\%$. 
Se observa que la temperatura del arrabio crece al aumentar en éste el contenido de silicio, pero la intensidad de crecimiento se hace más moderada de forma paulatina.

Estas conclusiones están en concordancia con la práctica operativa de los hornos altos. Sin embargo, los valores calculados para la temperatura del arrabio, según la ec. [2], resultan notablemente inferiores a los experimentales. Esta divergencia se explica no sólo por las estimaciones supuestas en el cálculo para la obtención del equilibrio de las reacciones de reducción del silicio, sino también por las diferentes influencias de una serie de factores tecnológicos durante los procesos de calentamiento de los productos de la fusión y de la reducción del hierro.

En las refs. (6 y 7) se estudian las influencias de los factores tecnológicos y las de las condiciones de fusión en función de la temperatura del arrabio y de su contenido de silicio.

En la medida en que se ha perfeccionado la tecnología de operación del horno alto (mejoras en la preparación de las materias primas, disminución del volumen de escoria, optimización de su composición, aumentos de la presión del viento y de los gases en el tragante, ICP, así como aumento del volumen útil de los hornos), se ha observado una tendencia al aumento de la temperatura del arrabio.

$\mathrm{Al}$ variar la temperatura en la zona de toberas, se puede regular la cantidad de $\mathrm{CO}$ que se forma a partir del $\mathrm{SiO}_{2}$ de las cenizas del combustible según $\left(\mathrm{SiO}_{2}\right)+2 \mathrm{C} \rightarrow[\mathrm{Si}]+2 \mathrm{CO}$ e influir en la velocidad de reducción a $\mathrm{SiO}$ de la sílice contenida en la escoria (8).

La limitación de la sublimación del $\mathrm{SiO}_{2}$ a $\mathrm{SiO}$ puede ser provocada por ciertos factores, tales como:

- Disminución de la actividad del $\mathrm{SiO}_{2}$ como resultado del aumento tanto de la basicidad de la carga como del contenido de $\mathrm{MgO}$.

- Disminución de la presión parcial del SiO al disminuir la temperatura delante de las toberas.

- Aumento de la presión parcial del CO debido al incremento de la presión en el tragante.

- Incremento de la productividad del horno como resultado del aumento de la velocidad de goteo de los productos líquidos de la fusión y disminución de su tiempo de retención en la zona cohesiva.

- Inyección por toberas de CP, mineral de hierro y fundentes (especialmente magnésicos).

Si se logra controlar el proceso del horno de tal forma que el silicio pase al arrabio sólo a partir del $\mathrm{SiO}$ procedente de las cenizas del combustible que se quema en toberas, entonces se podrá obtener arrabio con $0,1 \%$ Si y temperatura de $1.500{ }^{\circ} \mathrm{C}$.
Actualmente, algunos hornos altos japoneses que trabajan con carga de alta calidad, utilizan sistemas automatizados y siguen las mencionadas recomendaciones, fabrican arrabio bajo en silicio, con contenidos mínimos de azufre, a $1.500{ }^{\circ} \mathrm{C}$. Por ejemplo, durante más de 11 meses, todos los hornos altos de Mizushima Steel Works (Kawasaki Steel Corp.) han operado con contenidos de silicio en el arrabio del orden de 0,2\%. El contenido récord de silicio en el arrabio, para un período prolongado de operación, se alcanzó en el HA-5 de Fukuyama Steel Works (Nippon Kokan Corp.) con una media anual de $0,17 \%$ (8).

Por tanto, la regulación de determinadas variables del proceso puede garantizar ventajas en las variaciones del calentamiento químico o físico del arrabio.

Sin embargo, desde el punto de vista de la creación del SAD para el régimen térmico del horno, una conclusión importante es que, en cualquiera de las condiciones concretas, la relación entre la temperatura del arrabio y su contenido de silicio debe ser segura y fiable. Si varían estas condiciones, será necesario realizar la corrección de esta dependencia.

Será posible un conocimiento seguro del estado térmico del horno y su control efectivo cuando los parámetros que lo caracterizan (temperatura del arrabio y contenido de silicio) estén unidos en un solo parámetro complejo. Por ello, no es útil emplear la entalpía, debido a que una misma cantidad de calor tiene diferente valoración según los distintos potenciales caloríficos. La capacidad de trabajo del calor se determina por la magnitud de la exergía térmica $\left(E_{\mathrm{t}}\right)$ que considera el nivel de temperaturas del proceso (9).

Esta magnitud está constituida por las exergías química y física de la sustancia, según la ecuación:

$$
E_{\mathrm{t}}=E_{\mathrm{q}}+E_{\mathrm{f}}
$$

La exergía física del arrabio se calcula con ayuda de la siguiente ecuación (10):

$$
\begin{gathered}
E_{\mathrm{f}}=C_{1}\left(T_{\mathrm{f}}-T_{0}\right)+Q+C_{2}\left(T_{\mathrm{a}}-T_{\mathrm{f}}\right)-T_{0} \\
\left(C_{1} \ln \frac{T_{\mathrm{f}}}{T_{0}}+\frac{Q}{T_{\mathrm{f}}}+C_{2} \ln \frac{T_{\mathrm{a}}}{T_{\mathrm{f}}}\right.
\end{gathered}
$$

donde:

$E_{\mathrm{f}}=$ Exergía física, $\mathrm{kJ}$.

$C_{1}=$ Capacidad calorífica del arrabio en el intervalo $T_{0}-T_{\mathrm{f}}, \mathrm{kJ} / \mathrm{kg} \cdot \mathrm{K}$

$C_{2}=$ Capacidad calorífica del arrabio en el intervalo $T_{\mathrm{f}}-T_{\mathrm{a}}, \mathrm{kJ} / \mathrm{kg} \cdot \mathrm{K}$ 
$T_{\mathrm{a}}=$ Temperatura del arrabio, $\mathrm{K}$.

$T_{\mathrm{O}}=$ Temperatura ambiente, $\mathrm{K}$.

$T_{\mathrm{f}}=$ Temperatura del arrabio en la colada, $\mathrm{K}$.

$Q=$ Calor de fusión del arrabio, $\mathrm{kJ} / \mathrm{kg}$.

La exergía química del arrabio se determina mediante la ecuación:

$$
E_{\mathrm{q}}=Q_{\mathrm{i}} \cdot e x_{\mathrm{i}}
$$

donde:

$E_{\mathrm{q}}=$ Exergía química, $\mathrm{kJ}$.

$e x_{\mathrm{i}}=$ Exergía química específica de $i$ componentes en el arrabio, $\mathrm{kJ} / \mathrm{kg}$.

$Q_{\mathrm{i}}=$ Contenido en peso de $i$ componentes en el arrabio, $\mathrm{kg}$.

Los resultados de los cálculos según las ecs. [4] y [5] se incluyen en la tabla I.

Las variaciones químicas $\left(\Delta E_{\mathrm{q}}\right)$ y físicas $\left(\Delta E_{\mathrm{f}}\right)$ de la exergía, provocadas por las desviaciones del contenido de silicio en el arrabio $(\Delta[\mathrm{Si}])$ y su temperatura $(\Delta \mathrm{T})$, se determinan de la siguiente forma:

$$
\begin{array}{r}
\Delta E_{\mathrm{q}=0,01 e x_{\mathrm{Si}} \Delta[\mathrm{Si}]} \\
\Delta E_{\mathrm{f}}=C_{2}\left(\Delta T+T_{0} \ln \frac{T_{\mathrm{a}}-\Delta T}{T_{0}}\right)
\end{array}
$$

donde:

$e x_{\mathrm{Si}}=$ Exergía química específica del silicio en el arrabio $(21.824 \mathrm{~kJ} / \mathrm{kg})$.

La suma de las magnitudes mencionadas representa la desviación de la exergía térmica $\Delta E=\Delta E_{\mathrm{q}}$ $+\Delta E_{\mathrm{f}}$ y constituye un criterio complejo del estado térmico del crisol del horno. Por su magnitud, se pueden valorar las variaciones del calentamiento del crisol y realizar las acciones necesarias de control y regulación (11).

\section{DESARROLLO DE UN ALGORITMO DE CONTROL PARA EL RÉGIMEN TÉRMICO DEL CRISOL}

El control del estado térmico del crisol del horno alto se emplea como un parámetro de respuesta rápida para regular la tasa de ICP, y también como criterio de calidad del calentamiento del crisol, las desviaciones de la exergía térmica del arrabio y de sus componentes.

En la figura 3 se detalla el ordinograma que implementa el algoritmo de control que comanda el SAD. Ha sido desarrollado en un microcomputador cuya plataforma $\mathrm{Hw}$ está basada en un microprocesador análogo al i486 de Intel. Este microcomputador posee un sistema operativo DOS 5.3 con una memoria RAM de $16 \mathrm{Mb}$ y un disco duro de 640 $\mathrm{Mb}$; también contiene un amplio surtido de módulos que enlazan distintos periféricos, tipo captadores comunicaciones vía Ethernet, reguladores, etc, según se contempla en el diagrama de bloques de la figura 4.

Existe un módulo de adquisición de datos cuyo núcleo es un convertidor analógico-digital para bus AT, que recibe las señales, tanto analógicas como digitales de diferentes captadores y transductores, como la temperatura del arrabio durante la colada. Otro módulo de comunicaciones, en entorno Ethernet, garantiza las mismas (datos y órdenes) entre este microcomputador y otros computadores de procesos tanto remotos como centralizados (transmiten, por ejemplo, los resultados de los análisis químicos del arrabio y de la escoria). Otro módulo de conversión analógico-digital sirve para transmitir órdenes a los actuadores. Existen, asimismo, módulos exteriores de ampliación de memoria (tanto RAM como EPROM).

El sistema trabaja de la forma siguiente (11). Se mide el caudal de CP inyectado al horno. Durante el período de colada se interrumpe la ICP, y el sistema pasa a la medición de temperaturas del arrabio.

TABLA I.- Parámetros energéticos del estado térmico del crisol

TABLE I.- Energy parameters of the thermal state in the hearth

\begin{tabular}{|c|c|c|c|c|c|c|}
\hline \multirow{2}{*}{$\begin{array}{l}\text { Silicio en el } \\
\text { arrabio, \% }\end{array}$} & $\begin{array}{c}\text { Temperatura del } \\
\text { arrabio, }{ }^{\circ} \mathrm{C}\end{array}$ & \multicolumn{3}{|c|}{ Exergía, $\mathrm{kJ} / \mathrm{kg}$} & \multicolumn{2}{c|}{ Proporción, \% } \\
\cline { 2 - 7 } & $E_{\mathrm{q}}$ & $E_{\mathrm{f}}$ & $E_{\mathrm{t}}$ & $E_{\mathrm{q}}$ & $E_{\mathrm{f}}$ \\
\hline 0,4 & 1.380 & 7.926 & 890 & 8.816 & 89,9 & 10,1 \\
0,5 & 1.400 & 7.946 & 905 & 8.851 & 89,8 & 10,2 \\
0,6 & 1.420 & 7.971 & 918 & 8.889 & 89,6 & 10,4 \\
0,7 & 1.440 & 7.993 & 934 & 8.927 & 89,5 & 10,5 \\
0,8 & 1.460 & 8.015 & 949 & 8.964 & 89,4 & 10,6 \\
0,9 & 1.480 & 8.037 & 963 & 9.000 & 89,3 & 10,7 \\
1,0 & 1.500 & 8.058 & 978 & 9.036 & 89,2 & 10,8 \\
\hline
\end{tabular}




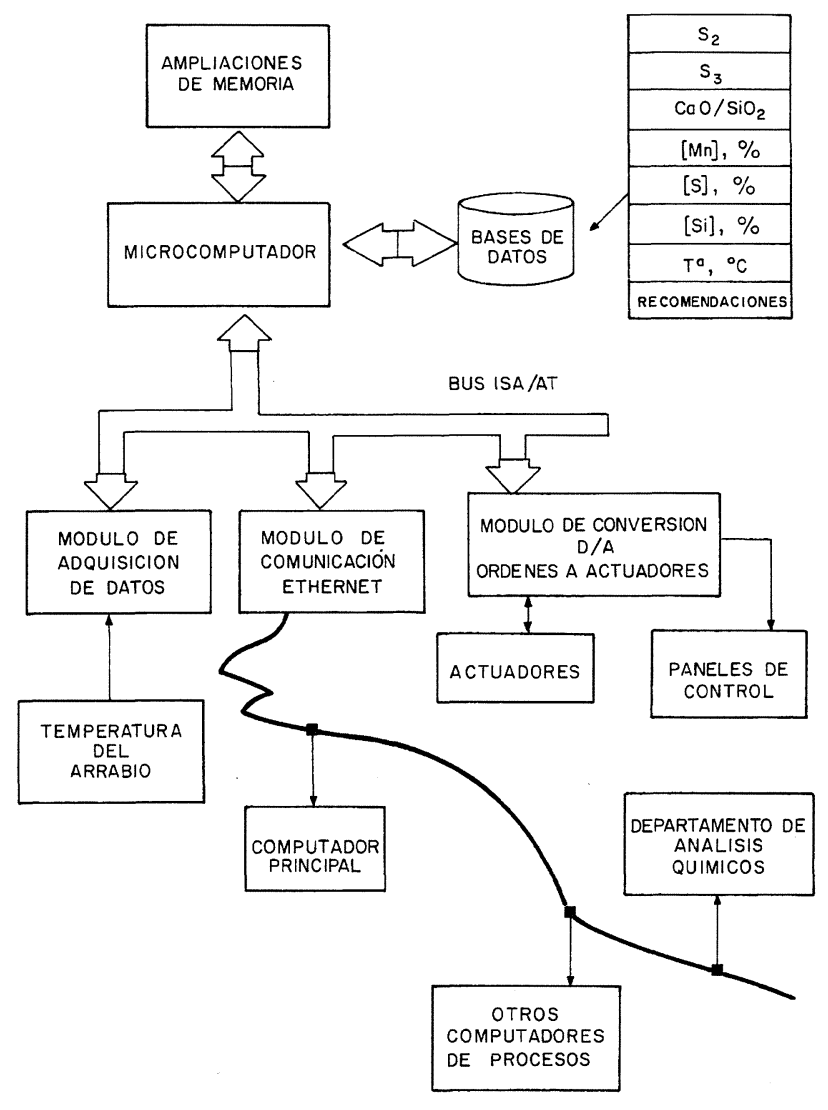

FIG. 3.- Diagrama de bloques de la plataforma $\mathrm{Hw} / \mathrm{Sw}$ del SAD.

\section{FIG. 3.-SAD Hw/Sw platform block diagram.}

La señal analógica del sensor fotopirométrico, restaurada y amplificada, es trasmitida al módulo de adquisición de señales, donde se eliminan las oscilaciones de alta frecuencia, obteniéndose así los valores instantáneos de temperatura. Se promedian estos valores cada 3 min durante el tiempo de colada, y se comparan con unos patrones (tablas cifradas) de operación contenidos en una base de datos relacional tipo Oracle, con lo que al término de la colada se determina la temperatura característica del arrabio.

Los datos sobre el contenido de silicio en el arrabio se transmiten al computador desde el laboratorio de análisis químico por línea Ethernet. Los valores característicos de la temperatura del arrabio y su contenido de silicio se emplean en el algoritmo para calcular el conjunto complejo del estado térmico. Los límites permisibles de variaciones de la exergía térmica, que corresponden al nivel térmico normal, son 350-450 kJ/kg. La desviación mínima de $\Delta E$, que requiere la acción de control selecciona$\mathrm{da}$, es $60 \mathrm{~kJ} / \mathrm{kg}$.

Al comparar los valores químicos, físicos y térmicos de la exergía del arrabio obtenidos con los almacenados anteriormente en la base de datos, el sistema analiza las tendencias y el ritmo de las variaciones del estado térmico del crisol y, en caso de necesidad, da recomendaciones al operador del panel de control para variar el caudal de inyección del CP.

El sistema de regulación operativa del régimen térmico de la operación del horno alto descrito se utiliza desde hace cinco años en el HA-1 de la planta siderúrgica de Donetsk, el sistema muestra seguridad y sencillez en el servicio, y fiabilidad en los resultados entregados. Los sistemas empleados garantizan la disminución de oscilaciones en los contenidos de silicio y azufre en el arrabio, lo que permite mejorar su calidad y economizar $4-5 \mathrm{~kg}$ de coque/t de arrabio.

Este sistema supone la posibilidad de unión con el sistema elaborado para la distribución del CP y oxígeno por toberas. El SAD descrito realizará las funciones jerarquizadas de nivel superior, determinando el caudal total de CP inyectado al horno.

En este SAD del estado térmico del crisol, al variar el caudal de CP inyectado, se logra operativamente la reducción de la entrada de calor por unidad de arrabio. Sin embargo, en este caso, se alteran otros parámetros tecnológicos de la fusión, tales como: temperaturas teórica y real de la combustión, basicidad de la escoria y volumen de gases del crisol.

Es posible la eliminación de estas incidencias desfavorables del régimen de marcha mediante la inyección simultánea en el crisol de CP y de otros aditivos (12). Una de estas medidas compensatorias consiste en inyectar $\mathrm{CP}$ mezclado con materiales férreos (MF). Al inyectar MF oxidados, tienen lugar reacciones del tipo:

$$
\begin{aligned}
& 2 \mathrm{FeO}+[\mathrm{Si}]=2[\mathrm{Fe}]+\left(\mathrm{SiO}_{2}\right) \\
& \mathrm{Fe}_{3} \mathrm{O}_{4}+2[\mathrm{Si}]=3[\mathrm{Fe}]+2\left(\mathrm{SiO}_{2}\right)
\end{aligned}
$$

que dan lugar a un aumento de la basicidad de la escoria, debido a la ganga introducida por estos materiales. Además, la reducción directa de los óxidos de hierro en la zona de toberas disminuye la temperatura de los gases existentes en esta zona.

Como las variaciones de caudal de MF son contrarias a las variaciones del caudal de $\mathrm{CP}$, se puede establecer una relación entre sus desviaciones con la que se garantice la constancia de la basicidad de la escoria y se dé lugar a variaciones mínimas en la temperatura de los gases en zona de toberas.

Con la determinación de la acción reguladora tanto del CP, en correspondencia con la ec. [1], como del MF, en correspondencia con su composición y el grado de desarrollo de las reacciones [8] y [9], se podrá tener en cuenta la influencia del contenido de silicio en el arrabio.

Se ha elaborado en el horno alto otro procedimiento de regulación operativa conjunta del 


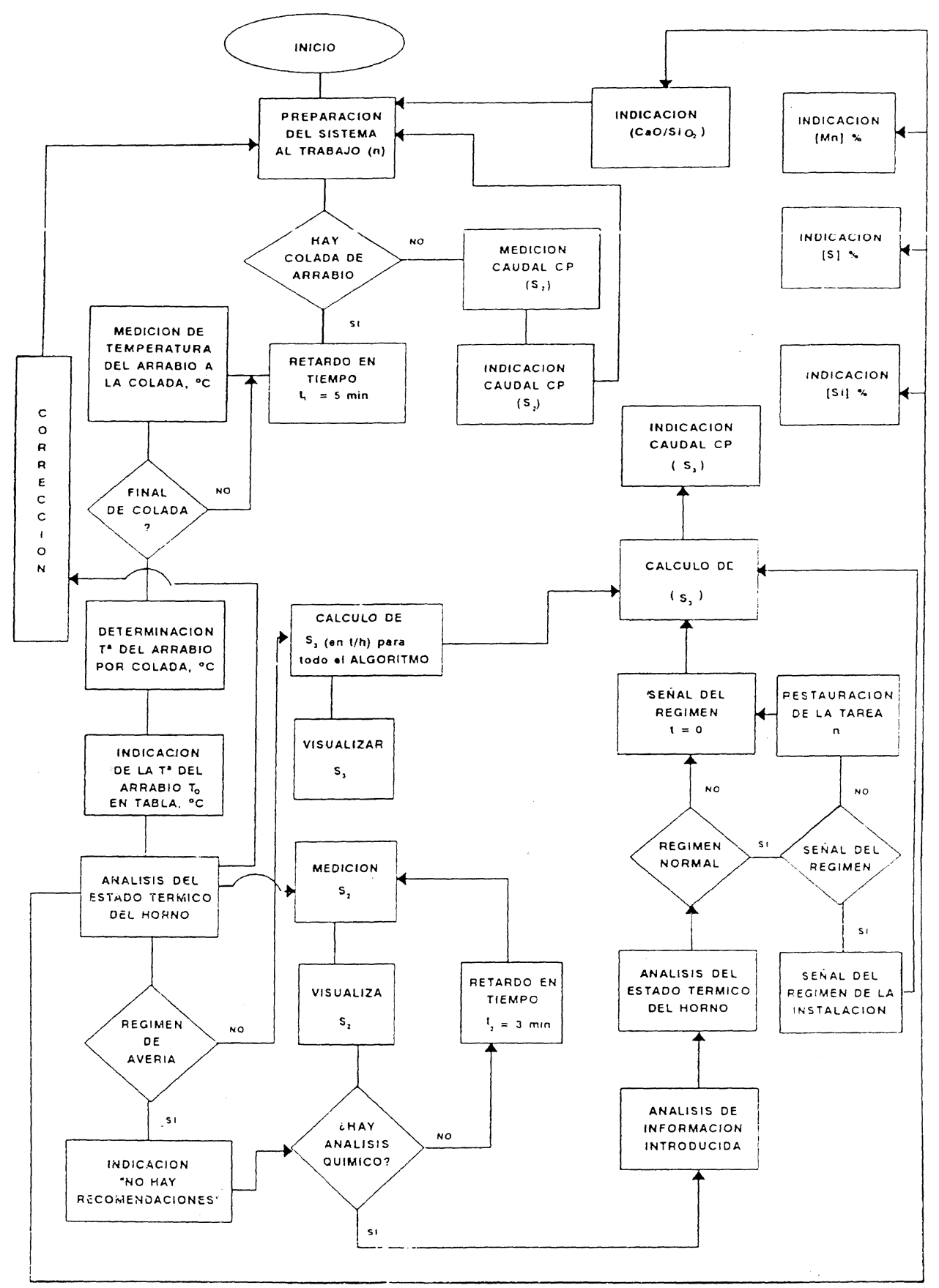

FIG. 4.- Ordinograma del algoritmo de control. $S_{2}$ : caudal de CP recomendado. $S_{3}$ : caudal de CP real. $T_{\mathrm{c}}$ : temperatura característica del arrabio en la colada.

FIG. 4.- Control algorithm flowchart. $\mathrm{S}_{2}$ : recommended PC flow. $\mathrm{S}_{3}$ : real PC flow. $\mathrm{T}_{\mathrm{c}}$ : characteristic temperature of pig iron in the casting.

calentamiento del crisol con la variante de inyección simultánea de CP con adiciones intensificadoras, en particular, con nitratos y dolomía (13).

La introducción de estos aditivos en la composición de la mezcla aumenta la concentración de agentes oxidantes $\left(\mathrm{O}_{2}\right.$ y $\left.\mathrm{CO}_{2}\right)$ en la zona en que comienza el proceso de combustión, lo que acelera la gasificación del CP debido al incremento de su superficie específica. Es conocido que, en condicio- nes reales, la superficie específica del $\mathrm{CP}$ está notablemente reducida por la formación de aglomerados resistentes de sus propias partículas. El desprendimiento simultáneo de los componentes volátiles de estas sustancias y de los volátiles del CP facilita la destrucción de los mencionados aglomerados y, por tanto, el aumento de la superficie específica del combustible hasta una magnitud límite de 0,2-0,5 $\mathrm{m}^{2} / \mathrm{g}$. 
Los resultados experimentales obtenidos mediante un estudio con un equipo de análisis termogravimétrico, de adiciones de $\mathrm{Ca}\left(\mathrm{NO}_{3}\right)_{2}$ y dolomía, $\mathrm{CaMg}\left(\mathrm{CO}_{3}\right)_{2}$, se incluyen en la tabla II. Estos ensayos, realizados con calentamiento hasta 1.000 ${ }^{\circ} \mathrm{C}$ y velocidades de $20{ }^{\circ} \mathrm{C} / \mathrm{min}$, muestran que, para 5 y $10 \%$ de $\mathrm{Ca}\left(\mathrm{NO}_{3}\right)_{2}$ en la mezcla, el tiempo de combustión se reduce en 14,2 y $28 \%$, respectivamente; para adiciones de 5 y $10 \%$ de dolomía también se observa la reducción del tiempo de combustión, pero en una menor proporción. Se debe destacar que el tiempo de desprendimiento de las sustancias volátiles, por el contrario, crece en una pequeña proporción, debido al incremento en 6-9 \% de la cantidad total de volátiles que se desprenden.

En conclusión, dado que estas sustancias contienen oxígeno complementario, se puede compensar tanto la temperatura adiabática de combustión como el volumen de gases del crisol (y consecuentemente la velocidad de descenso de la carga) mediante la regulación de los componentes de la mezcla, todo lo cual ocurre por el control del estado térmico del crisol mediante la tasa de ICP.

La esencia del procedimiento mencionado consiste en que, en caso de enfriamiento del crisol, por cada $\mathrm{O}, 1 \%$ de disminución del contenido de silicio en el arrabio se puede incrementar el caudal de mezcla combustible inyectada en $5-20 \mathrm{~kg} / \mathrm{t}$ de arrabio con una disminución simultánea de la relación $\mathrm{CP} /$ nitratos de 0,01-0,05. En caso de calentamiento del crisol, o sea aumento del contenido de silicio en el arrabio, se puede realizar una operación inversa.

Se ha desarrollado otro método con el fin de regular el calentamiento del crisol, que realiza la inyección de dos tipos de carbones, con contenidos de sustancias volátiles bajos (hullas magras, antracitas) y altos (energéticos). Las hullas magras y la antracita varían el volumen de gases y la temperatu- ra de combustión en las toberas en menor grado que los carbones energéticos. Por tanto, si es necesario realizar la regulación del calentamiento del crisol se puede variar no sólo la tasa total de ICP, sino también la relación entre estos carbones, lo que permite compensar las desviaciones de los parámetros tecnológicos mencionados.

\section{CONCLUSIONES}

- La creación de SAD locales, tomando como base los mini y microcomputadores, y la construcción de sistemas jerárquicos de niveles múltiples, tomando como base los mismos, es una de las vías con más perspectivas en el desarrollo del control automático del proceso del horno alto.

- La rápida respuesta de la ICP como combustible complementario, con influencias mínimas en la temperatura, composición y calidad de los gases de la zona de toberas, determina la efectividad de utilización del CP para ejercer una acción rápida sobre el estado térmico del crisol del horno alto.

- La dependencia entre la temperatura del arrabio y su contenido de silicio es segura y fiable para cualquiera de los parámetros del proceso, pero también influyen las condiciones en las que se realiza la fusión. La regulación de ciertos factores tecnológicos individuales puede garantizar variaciones ventajosas en el calentamiento químico o físico del arrabio.

- Se ha elaborado un SAD local para el régimen térmico del crisol del horno alto con variación de la tasa de ICP. La desviación de la exergía

TABLA II.- Influencia de las adiciones de $\mathrm{Ca}\left(\mathrm{NO}_{3}\right)_{2}$ y dolomía $\mathrm{CaMg}\left(\mathrm{CO}_{3}\right)_{2}$ en la combustión del CP TABLE II.-Additions of $\mathrm{Ca}\left(\mathrm{NO}_{3}\right)_{2}$ and dolomite, $\mathrm{CaMg}\left(\mathrm{CO}_{3}\right)_{2}$, on PC combustion

\begin{tabular}{|l|c|c|c|c|c|}
\hline Composición de mezclas & $\begin{array}{l}\text { Tiempo total de } \\
\text { combustión, min }\end{array}$ & $\begin{array}{l}\text { Duración relativa de las etapas } \\
\text { de combustión, \% }\end{array}$ & $\begin{array}{l}\text { Temperatura de } \\
\text { ignición del coque } \\
\text { residual, }{ }^{\circ} \mathrm{C}\end{array}$ & $\begin{array}{l}\text { Temperatura } \\
\text { final de fusión, } \\
{ }^{\circ} \mathrm{C}\end{array}$ \\
\cline { 3 - 5 } & & $\begin{array}{l}\text { Desprendimiento } \\
\text { de volátiles }\end{array}$ & $\begin{array}{l}\text { Combustión } \\
\text { del coque } \\
\text { residual }\end{array}$ & & \\
\hline $\mathrm{CP}+2,5 \% \mathrm{Ca}\left(\mathrm{NO}_{3}\right)_{2}$ & 103,0 & 15,4 & 74,3 & 550 & 900 \\
$\mathrm{CP}+5 \% \mathrm{Ca}\left(\mathrm{NO}_{3}\right)_{2}$ & 104,4 & 14,6 & 73,9 & 560 & 915 \\
$\mathrm{CP}+10 \% \mathrm{Ca}\left(\mathrm{NO}_{3}\right)_{2}$ & 89,6 & 22,3 & 66,1 & 618 & 930 \\
$\mathrm{CP}+5 \%$ dolomía & 74,8 & 29,9 & 56,1 & 660 & 920 \\
$\mathrm{CP}+10 \%$ dolomía & 99,6 & 18,9 & 69,4 & 566 & 882 \\
\hline
\end{tabular}


térmica del arrabio de un nivel dado constituye un criterio del estado térmico. La explotación industrial del sistema garantiza la mejora de la calidad del arrabio y una economía de coque de 4-5 kg/t de arrabio.

- Se han elaborado métodos de regulación operativa del régimen térmico de fusión, que garantizan, conjuntamente con la reducción de la entrada de calor por unidad de arrabio elaborado, compensaciones de las desviaciones de temperatura y volumen de gases en la zona de toberas y de la basicidad de la escoria de acuerdo con las variaciones de las relaciones de los caudales de las mezclas combustibles inyectadas en el crisol.

\section{Agradecimiento}

Los autores agradecen al Ministerio de Educación y Ciencia (Ref. Sab. 92-0144 y Sab. 95-0151) (Ref. Sab. 95-0151). ECSC (CE) (Agreement No. 7210. AA/936). ECSC (CE) (Agreement No. 7210. AA/938) los apoyos económicos recibidos. Asimismo, a la Factoría Siderúrgica de Donetsk (Ucrania) la realización de los ensayos industriales.

\section{REFERENCIAS}

(1) Matsuda, K., Nakyama, M., Otsuka, Y. y Konishi, M. Proc. 1st Int. Congr. on Sci. and Tech. of Ironmaking. ISIJ. Sendai (Japón), 1994: 302-307.

(2) Peters, H. y Plüm, H.-D. Stahl Eisen, 114 (4), 1994: 39-46.

(3) Inkola, P., Karppinen, A. y Seppyanen, M. Stal, (9), 1994: 7-12.

(4) Babich, A., Yaroshevskil, S. y Mitasov, O. Steel URSS, 12 (5), 1982: 189-191.

(5) Babich, A., YaroshevskiI, S. y SkladanovskiI, E. Stal, (12), 1983: 10-14.

(6) Kaneko, K., Senoo, Y. y Kigushi, M. Kawasaki Steel Giho, 19 (13), 1987: 156-161.

(7) KIm, V.A. y Belyakov, N.N. Stal, (11), 1994: 15-20.

(8) FuruYa, S., NaKajima, R., Hattori, M., Shimomura, A. y YAMAOKA, Y. Steel Tech. Int., 1992: 27-30.

(9) Stepanov, V. Jimicheskaya energiya y eksergiya veshestv., Nauka. Novosibirsk, 1985.

(10) Babich, A., Borodin, A. y Skladanovskit, E. Pat. URSS núm., 1668402 (1991).

(11) Babich, A., Semenijin, V., Stepanov, V. y SkaldanovsKII, E. Bol. Inst. Chermetinformatsiya Metallurgiya, (5), 1991: 67-68.

(12) Babich, A., Yaroshevskit, S., Garcia, L., Formoso, A., Cores, A., Isidro, A. y Ferreira, S. Rev. Metal. Madrid, 32 (2), 1996: 103-116.

(13) Babich, A., YaroshevskiI, S., Formoso, A., Isidro, A., Ferreira, S., Cores, A. y Garcia, L. ISIJ Int., 36 (10), 1996: $1.250-1.258$. 